\title{
Remembering Sergio Colafrancesco (1957-2018) (Scientist and Friend)
}

\author{
Franco Giovannelli* \\ INAF - Istituto di Astrofisica e Planetologia Spaziali, Via del Fosso del Cavaliere, 100, 00133 \\ Roma, Italy \\ E-mail: Eranco.giovannellidinaf.it
}

\begin{abstract}
It is a poignant task for me to remember Sergio Colafrancesco in this international context, in part because he started his path here that allowed him to become a very well known scientist, He did so because of his magnificent talks which drew on his profound knowledge of physics. He was a great scientist and friend.

I do not want to enter in his scientific production which is largely demonstrated in his 621 published papers and thousands of citations. I prefer to remember Sergio in his many activities that I have the privilege of knowing, thanks to our long association and friendship.

I will use for this commemoration some material coming from A meeting in honor of Sergio Colafrancesco organized by Paolo de Bernardis and Silvia Masi on Novembre 2, 2018 at the Department of Physics of the Sapienza University of Roma with the contributions of Paolo de Bernardis \& Silvia Masi, John Carter, Franco Giovannelli, and Angelo Antonelli, and from the official memorial service A celebration of the life and work of Sergio Colafrancesco organized in Johannesburg at Hofmeyr House of the Wits University on June 14, 2019.
\end{abstract}

Multifrequency Behaviour of High Energy Cosmic Sources - XIII - MULTIF2019

3-8 June 2019

Palermo, Italy

\footnotetext{
* Speaker.
} 


\section{Introduction}

Sergio was born in Rocca d'Arce - a small village of 941 inhabitants located about 100 kilometres southeast of Roma in the province of Frosinone, Italy, on May 3, 1957 and passed away in Rocca d'Arce on September 30, 2018 (Fig. 1).

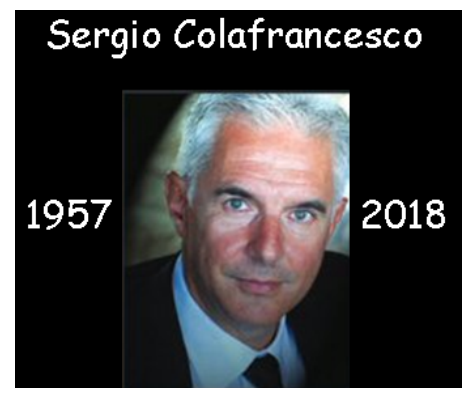

Figure 1: Sergio Colafrancesco: Scientist.

Sergio's main activities has been developed within four Institutions:

- University of Padua, Italy

- Osservatorio Astronomico Monte Porzio Catone, Italy (now INAF)

- ASI (Agenzia Spaziale Italiana), Italy

- University of Witwatersrand, Johannesburg, South Africa

\section{Sergio at the Beginning}

Sergio studied in Roma at the University "La Sapienza" receiving his Degree in Physics and then he obtained his PhD in Astronomy at the University of Padua, Italy.

Among several papers that appeared when he was in Padua, a couple of them - Space distribution of X-ray clusters of galaxies (Cavaliere \& Colafrancesco, 1988) and The mass function from local density maxima-groups and clusters of galaxies (Colafrancesco, Lucchin \& Matarrese, 1989) - caught my attention and suggested to me that I invite Sergio to Vulcano to attend my workshops.

These very well known biennial workshops were: Frontier Objects in Astrophysics and Particle Physics, and Multifrequency Behaviour of High Energy Cosmic Sources.

The two workshops functioned as a launching pad for Sergio, who became better known in the international scientific Community thanks to his works presented there. This allowed him to meet personally several colleagues with whom he began some fruitful collaborations (e,g. Blasi \& Colafrancesco, 1999; Beall et al., 2003; Colafrancesco, Dar, De Rújula, 2004).

\section{Sergio's Contribution to Vulcano Workshops}

I would like to recall Sergio's presence at our workshops to demonstrate how much he contributed to their success both from a scientific and critical points of view. To this end, in Fig. 
2, I report the first page of his Concluding Remarks of the Frascati Workshop 2001 on Multifrequency Behaviour of High Energy Cosmic Sources - IV" (Colafrancesco, 2002), and in Fig. 3 his Final Remarks of the Vulcano Workshop 2002 on "Frontier Objects in Astrophysics and Particle Physics - IX", where he made a statistical description of the scientific contributions presented there (Colafrancesco, 2003).

\title{
SERGIO COLAFRANCESCO
}

INAF - Osservatorio Astronomico di Roma

Via Frascati 33, I-00040 Monteporzio - ITALY

\begin{abstract}
1. Piecing together the puzzle: a personal view of the Vulcano meeting
One of the most interesting aspects of the results presented at this Meeting is, in my opinion, the evidence of an undergoing cognitive process leading from the standard to a precision era in astrophysics.

This is evident from the large variety of physical phenomena discussed during the meeting and from the attempt to apply the Physics that is being tested here and now in our Solar System to different cosmic scales, wavelenghts and epochs.

Examples of such a process can be extracted from three subjects, among others, that have been widely addressed in this Meeting: $i$ ) galaxies, ii) clusters of galaxies and iii) cosmology.
\end{abstract}

Figure 2: First page of the concluding remarks of the Frascati Workshop 2001 "Multifrequency Behaviour of High Energy Cosmic Sources - IV" (Colafrancesco, 2002).

The impressive concluding remarks were reported by Sergio at the end of the Frascati Workshop 2013 in which he gave "Some Personal Conclusions about the logical development of the workshop: One of the main aspects of this Meeting is its capacity to induce global discussions on some of the most important challenges in astrophysics and cosmology. These challenges address the nature of fundamental questions in the physics of the universe, the frontier of exa-scale data mining and analysis and the technological challenges related to the construction of the largest astronomical facilities in the next decade (Colafrancesco, 2014).

\section{The many-sided genius of Sergio}

It is difficult to list all the activities of Sergio. However, as example, Fig. 4 reports the experiments in which Sergio was involved, giving suggestions for programming observations, for the scientific use of data, and sometimes for the design of experiments.

His participation was particularly important in the OLIMPO and MILLIMETRON experiments.

The OLIMPO experiment uses the Cosmic Microwave Background (CMB) as a backlight to study the largest structures in the Universe. It is optimized to detect the inverse Compton scattering of CMB photons crossing clusters of galaxies. This is also known as the Sunyaev-Zeldovich effect (SZE). Using the SZE, OLIMPO can detect very distant galaxy clusters, which could not be detected in other ways. Additional science targets of OLIMPO are the spectral-spatial anisotropies of the CMB and neutrino masses from high-angular resolution maps of the CMB. 
The MILLIMETRON space observatory (Spektr-M) is a project of Astro Space Center of Lebedev Physical Institute, Russian Academy of Sciences (ASC LPI). It will be a 10-meter space telescope designed to study various objects in the Universe at millimeter and infrared wavelengths from 0.07 to $10 \mathrm{~mm}$. Scientific program of Millimetron Space Observatory is aimed to solve a set of the most important and breakthrough scientific problems in the field of modern astrophysics and cosmology, namely a detailed study of black hole shadows, the search for wormholes, water trail within the Galaxy, and CMB spectral distortions. Fig. 6 shows a sketch of the early payload with

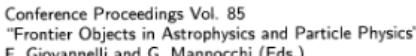

Conference Proceedings Vol. 85 "Frontier Objects in Astrophysics and Particle Physics" F. Giovannelli and G. Mannocchi (Eds.)
SIF, Bologna, 2003

\title{
Concluding remarks - III
}

\author{
S. Colafrancesco
}

INAF - Osservatorio Astronomico di Roma Via Frascati, 33 I-00040 Monteporzio (Roma) Italy

Email: cola@mporzio.astro.it

My concluding remarks will mainly deal with a (personal) statistical description of the scientific contributions to this Meeting and only marginally with their scientific content being the level of the many invited and contributed talks unequivocally evident.

This Meeting gave us the possibility to attend 65 talks divided in 8 scientific sessions as reported in Fig. 1. Two workshop directors organized the agenda of 40 Italian and 21 non-Italian scientists providing a full-time, multi-disciplinary interaction for the whole duration of the Meeting.

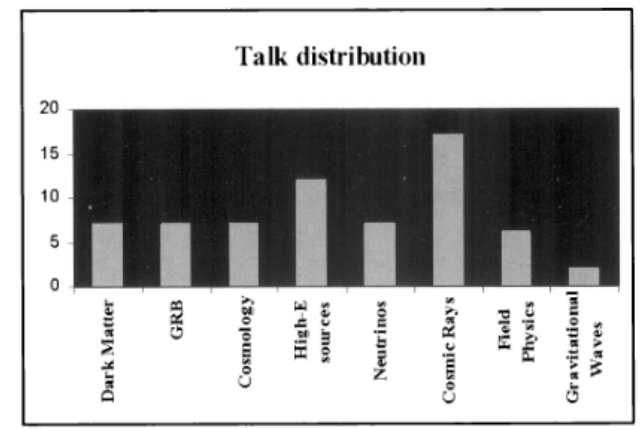

Figure 1: The distribution of the 65 talks at this Meeting divided in the 8 scientific sessions as labeled.

Figure 3: Concluding remarks of the Vulcano Workshop 2002 "Frontier Objects in Astrophysics and Particle Physics - IX" (Colafrancesco, 2003). 


\begin{tabular}{ll} 
H.E.S.S. & PLANCK \\
AGILE & CORE \\
MIGHTEE & XRT \\
CTA & SPIRE \\
SKA & SUZAKU \\
ATCA & INTEGRAL \\
SWIFT & WMAP \\
FERMI & OLIMPO \\
ATOM & EXTREMO/WFXRT \\
PRISM & CHANDRA \\
MILLIMETRON & BePPO/SAX \\
\hline
\end{tabular}

Figure 4: A list of experiments in which Sergio was involved.

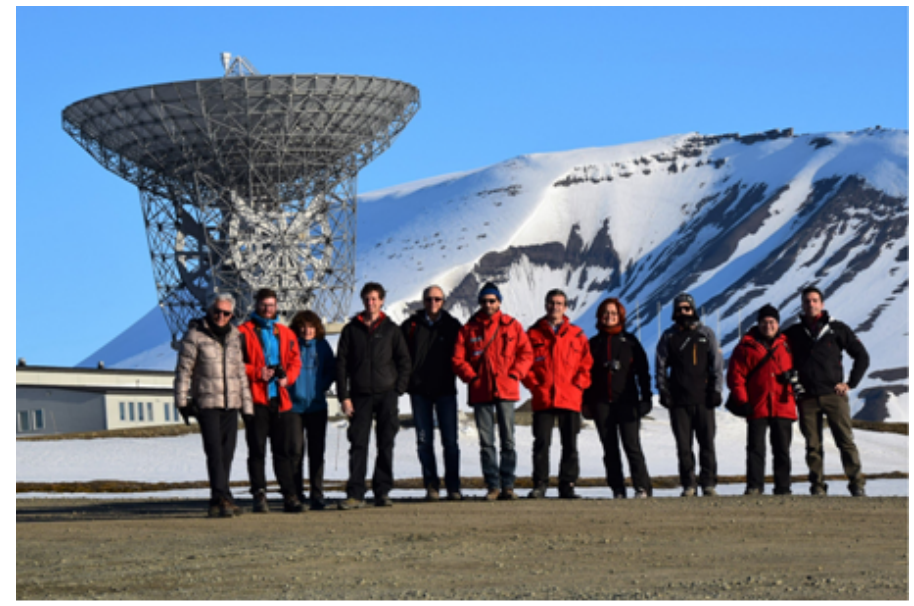

Sergio and the OLIMPO crew at EISCAT - Spitzbergen - OLIMPO launch campaign (June 2014) 1 week to work at the optimization of the observation targets and measurements plan
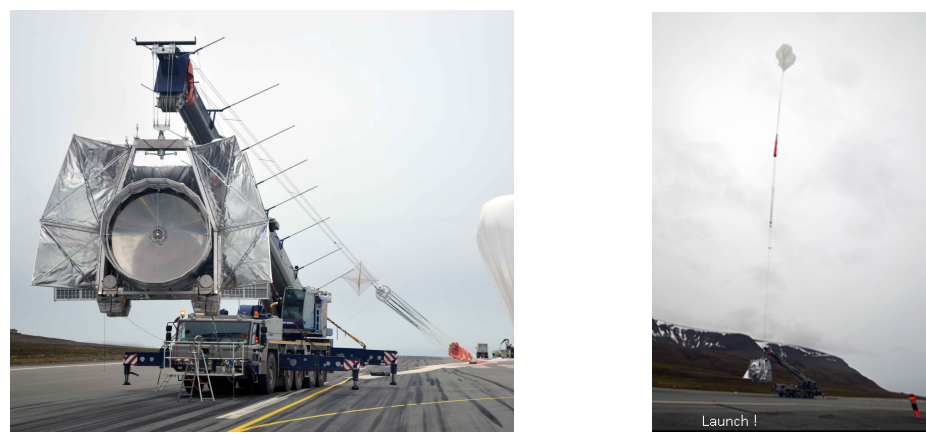

Figure 5: Upper panel: OLIMPO launch campaign (June 2014). Lower left panel: OLIMPO 2018 ready for Launch (Longyearbyen July 14th). Lower right panel: OLIMPO Launch. Images taken from the contribution of Paolo de Bernardis and Silvia Masi at the meeting in honor of Sergio Colafrancesco held in Roma, Sapienza University on November 2, 2018. 
the contribution from Sergio.

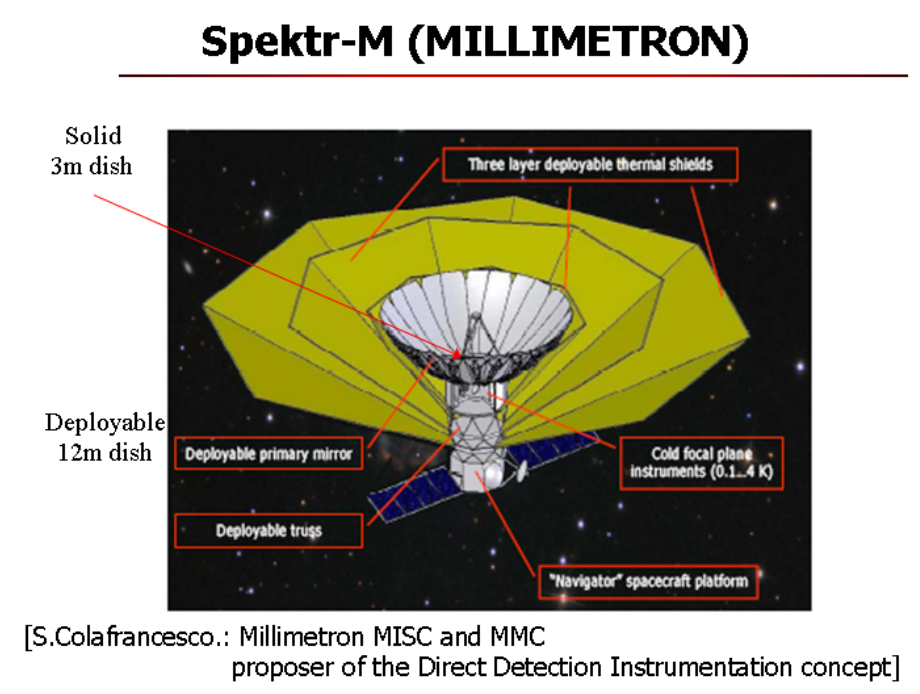

Figure 6: A sketch of Millimetron early payload.

Sergio was also interested in "Laboratory for Education \& Public Outreach (EPO) in Astronomy". He was particularly sensitive to the issue of investments that different countries make available for science. He was saddened by observing how little his country (Italy) put into the enterprise, as can be seen in Fig. 7 (Colafrancesco, 2004).

S. Colafrancesco: Laboratories for EPO in Astronomy

433

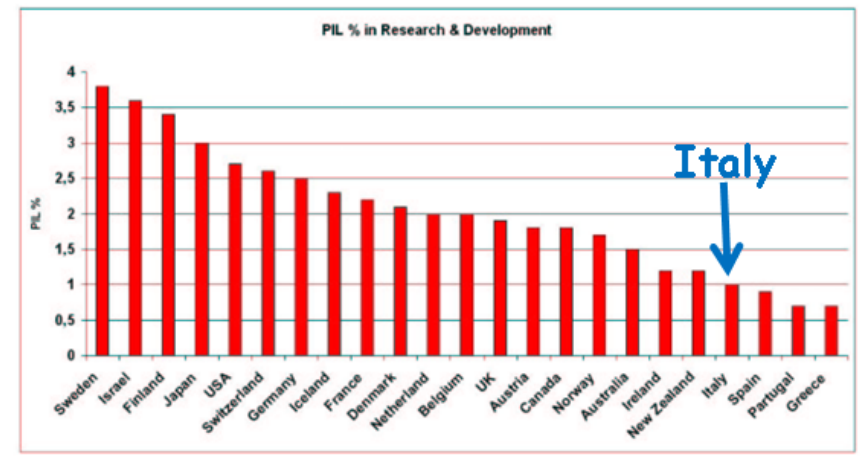

Fig. 1. The distribution of the 2003 PIL fraction which goes into research and technology for the 20 more developed countries. Data from United Nation Organization.

Figure 7: Laboratory for Education \& Public Outreach (EPO) in Astronomy (Colafrancesco, 2004).

Sergio was also interested in preserving telescopic plate archives in order to have memory of ancient astronomical events useful for novae, supernovae, and in general for variable cosmic sources. The left panel of Fig. 8 shows Sergio at the Plate Archive of Wits University.

He also gave important contributions to the progress of VHE astrophysics in South Africa. The central and right panels of Fig. 8 show Sergio at the "Inauguration of H.E.S.S. II", and a partial view of the H.E.S.S. II experiment, respectively. 

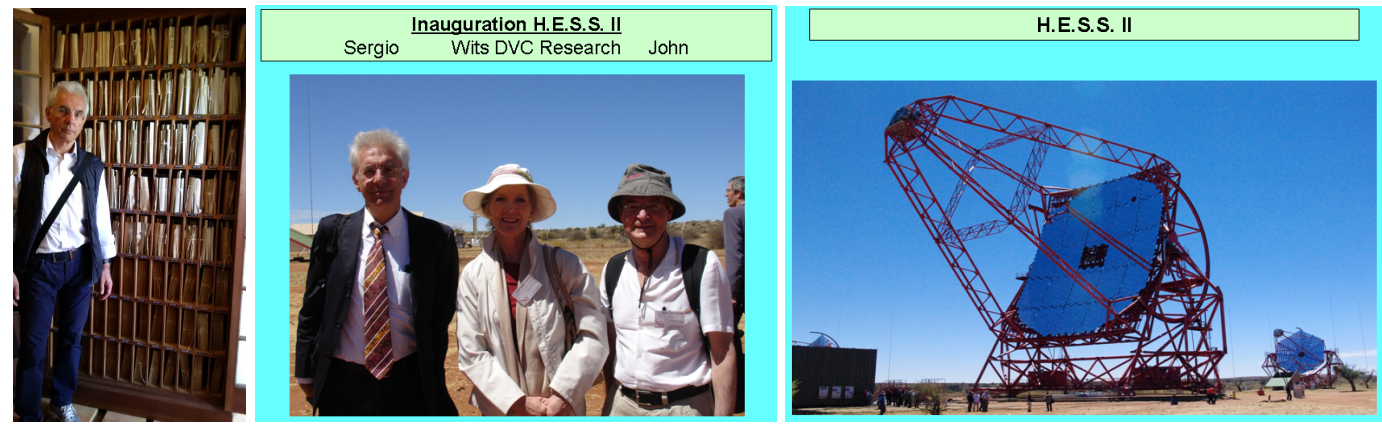

Figure 8: Left panel: Sergio at the plate archive of Wits University. Central panel: photo during the inauguration of H.E.S.S. II. Right panel: H.E.S.S. II experiment (images taken from the contribution of John Carter at the meeting in honor of Sergio Colafrancesco held in Roma, Sapienza University on November 2, 2018).

\section{Why Sergio moved to Johannesburg}

This is a delicate point in Sergio's story of which I am probably the only true witness. In 2010 Sergio and I enthusiastically organized a "Science with Millimetron" workshop to discuss all the news that this mission would bring to the knowledge of the physics of the Universe. The workshop was located in Mondello (Palermo, Italy). the left panel of Fig. 9 shows the poster of the workshop.

The talk prepared by Sergio - as scientist of ASI (Italian Space Agency) - was "Cosmology with the SZE".

But ASI forbade him to participate in the workshop he organized with my collaboration. Thus, I presented his talk, to the surprise of the audience. Also Igor Novikov was there together with the co-Is of the mission. They did not understand what was going on. Right panel of Fig. 9 shows the first slide of Sergio's talk.

This was a terrible blow that infuriated Sergio. Questa fu la goccia che fece traboccare il vaso that in English sounds This was the last straw that broke the camel's back!

Shortly after this, Sergio moved to WITS University in Johannesburg, South Africa.

At the WITS University, Sergio fully engaged in all of his scientific and human powers, thanks to the triumphant welcome that this University gave him. He created an astrophysics group with which he ventured into a multitude of activities that are schematically represented in Fig. 10.

To remember the magnificence of his slides I would like to mention a couple of them coming from two speeches made by Sergio during two workshops in 2012, as shown in Fig. 11. The papers about these arguments have been published (e.g. Colafrancesco, 2013; Ade et al. - Planck Collaboration, 2013a,b). 
I.M.C. Workshop 2010

Science with Millimetron

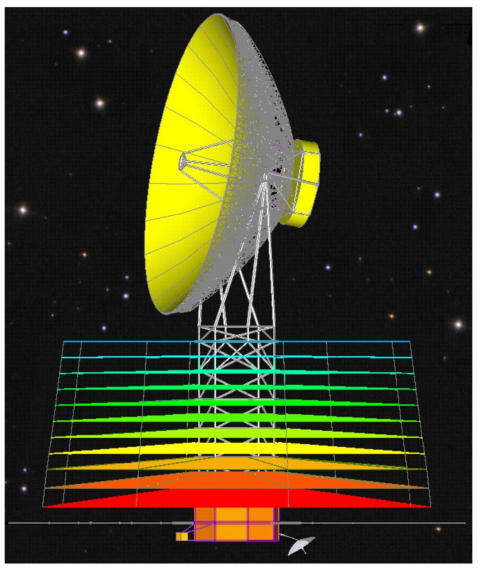

Palermo (Italy) $14^{\text {th }}-18^{\text {th }}$ June 2010

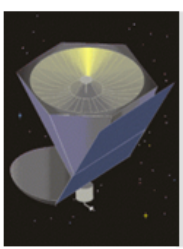

Cosmology with the SZE

Sergio Colafrancesco

Italian Space Agency (ASI)

Presented by

F. Giovannelli

Figure 9: Left panel: poster of the workshop Science with Millimetron organized by Sergio Colafrancesco and Franco Giovannelli. Right panel: the first slide of Sergio's talk presented by Franco Giovannelli.

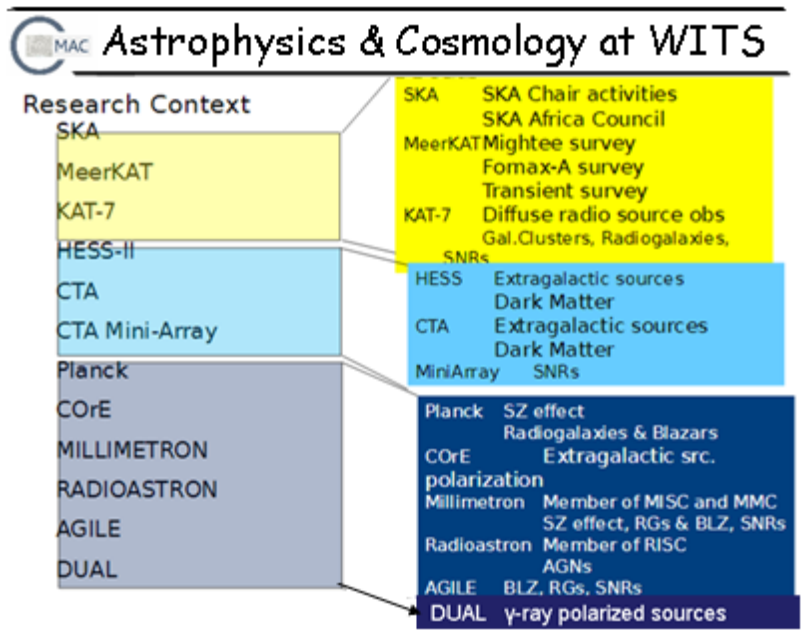

Figure 10: The numerous Sergio's activities at the WITS University.

\section{The many faces of Sergio's personality}

Figure 12 shows several photos that paint the many faces of Sergio's personality. Figure 13 shows some caricatures of Sergio about his comic vision of several events during our workshops.

\section{Sergio's candidature for the Grüber Foundation Prize}

At the end of 2017, the Grüber Foundation invited me to propose a nomination for the Grüber Prize 2018 for Cosmology. I proposed the candidacy of Sergio. For the rules of the Prize, the letter of presentation must be written by a person of an institution different from that of the proponent. I 


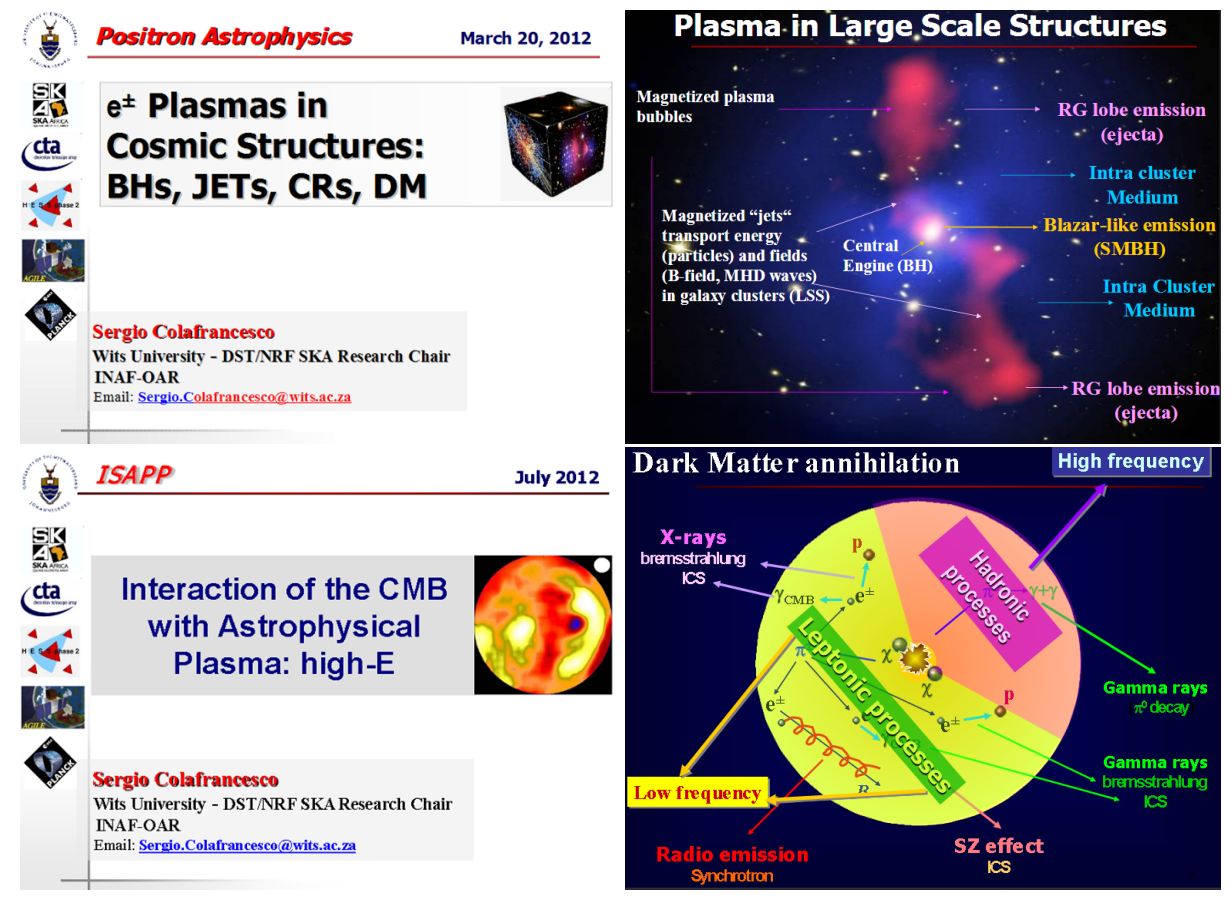

Figure 11: From left upper panel clockwise: Positron Astrophysics talk, Plasma in Large Scale Structure, Dark Matter Annihilation, ISAAP talk.

asked James Howard Beall to write it. Jim knew Sergio for many years from the Vulcano Meetings and their collaborations on papers. On that occasion, Sergio listed his five most important articles:

1. Sergio Colafrancesco, Stefano Profumo, Piero Ullio: Multi-frequency analysis of neutralino dark matter annihilations in the Coma cluster, 2006, A\&A 455 (1), 21-43.

2. Pasquale Blasi, Sergio Colafrancesco: Cosmic rays, radio halos and non-thermal X-ray emission in clusters of galaxies, 1999, Astroparticle Physics, 12 (3), 169-183.

3. Colafrancesco, Sergio, Lucchin, Francesco, Matarrese, Sabino: The mass function from local density maxima - Groups and clusters of galaxies, 1989, ApJ 345, 3C.

4. Colafrancesco, S., Marchegiani, P., Palladino, E.: The non-thermal Sunyaev-Zel'dovich effect in clusters of galaxies, 2003, A\&A 397, 27C.

5. Colafrancesco, S., Marchegiani, P., Emritte, M.S.: Probing the physics and history of cosmic reionization with the Sunyaev-Zel'dovich effect, 2016, A\&A 595A, 21C.

Among these five most important papers listed by Sergio, the number 3) is one of the two papers that pushed me to invite him for the first time in Vulcano. Thus, the scientific loop is objectively closed.

Left panel of Fig. 14 shows the heading of the letter for supporting Sergio for the Grüber Price for Cosmology 2018. Right panel of Fig. 14 shows the answer of the Grüber Foundation to me. 


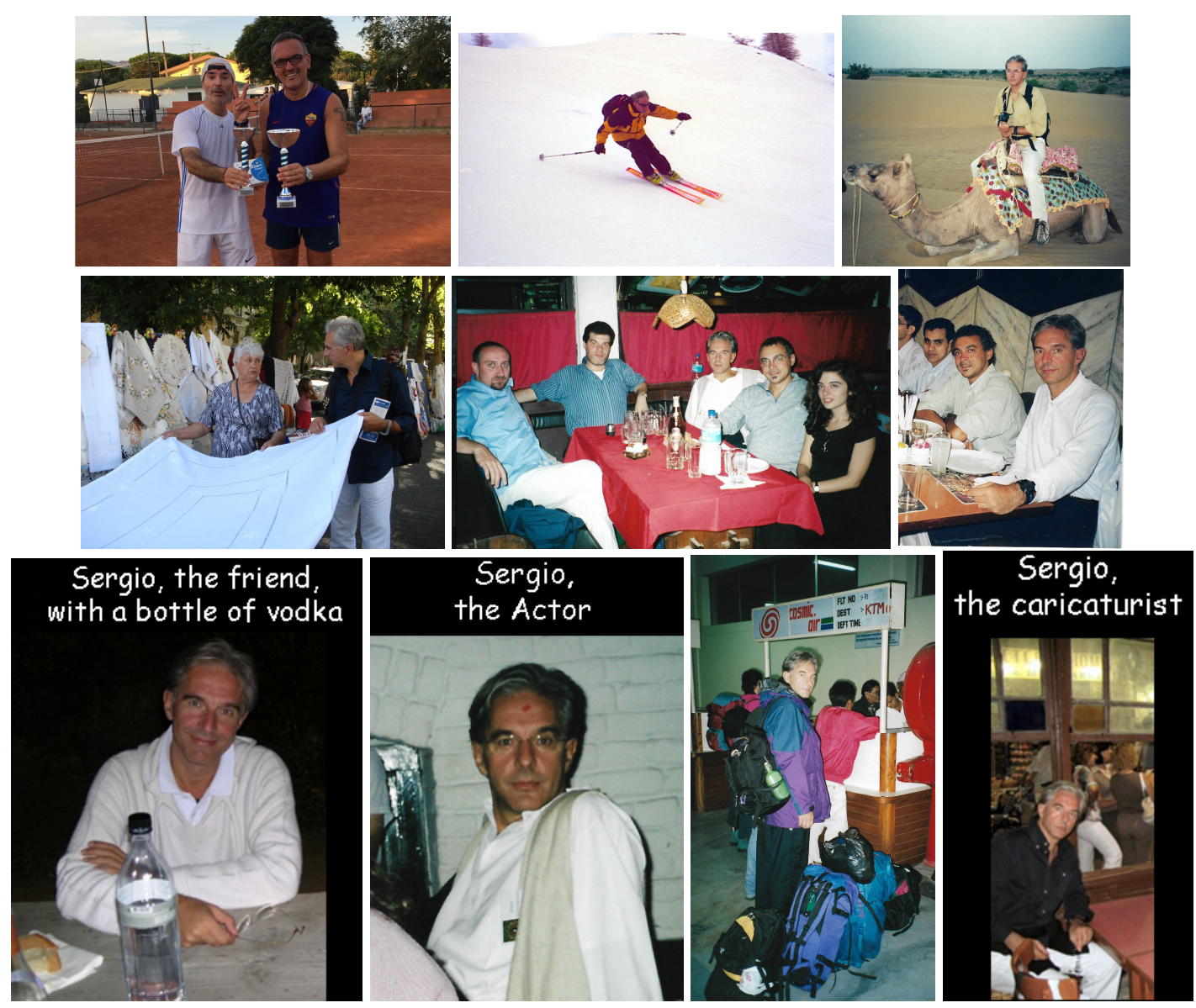

Figure 12: Upper panel from left to right: Sergio, the tennis player, Sergio, the skier, Sergio, the cameldriver. Middle panel from left to right: Sergio at the Odessa market by bargaining for the price (Credit: Franco Giovannelli, 2009), Sergio in Kathamandu, 2004. Lower panel from left to right: Sergio, the friend, Sergio, the actor, Sergio, the traveller, Sergio, the caricaturist (Images taken from the contribution of Angelo Antonelli at the meeting in honor of Sergio Colafrancesco held in Roma, Sapienza University on November 2, 2018).

\section{Sergio's latest message about the future of research in Cosmology}

Modern cosmology is more and more addressing questions of fundamental physics in the universe:

- What is the nature of Dark Matter?

- What is the origin of magnetic fields and HE cosmic rays in large-scale structures?

- How Dark Matter and cosmic rays influence the evolution of large-scale structures in the universe?

- And what are their feedback on smaller cosmological scales?

- Can we use galaxy clusters (and other cosmic structures) to unveil the fundamental physics of the early universe? 


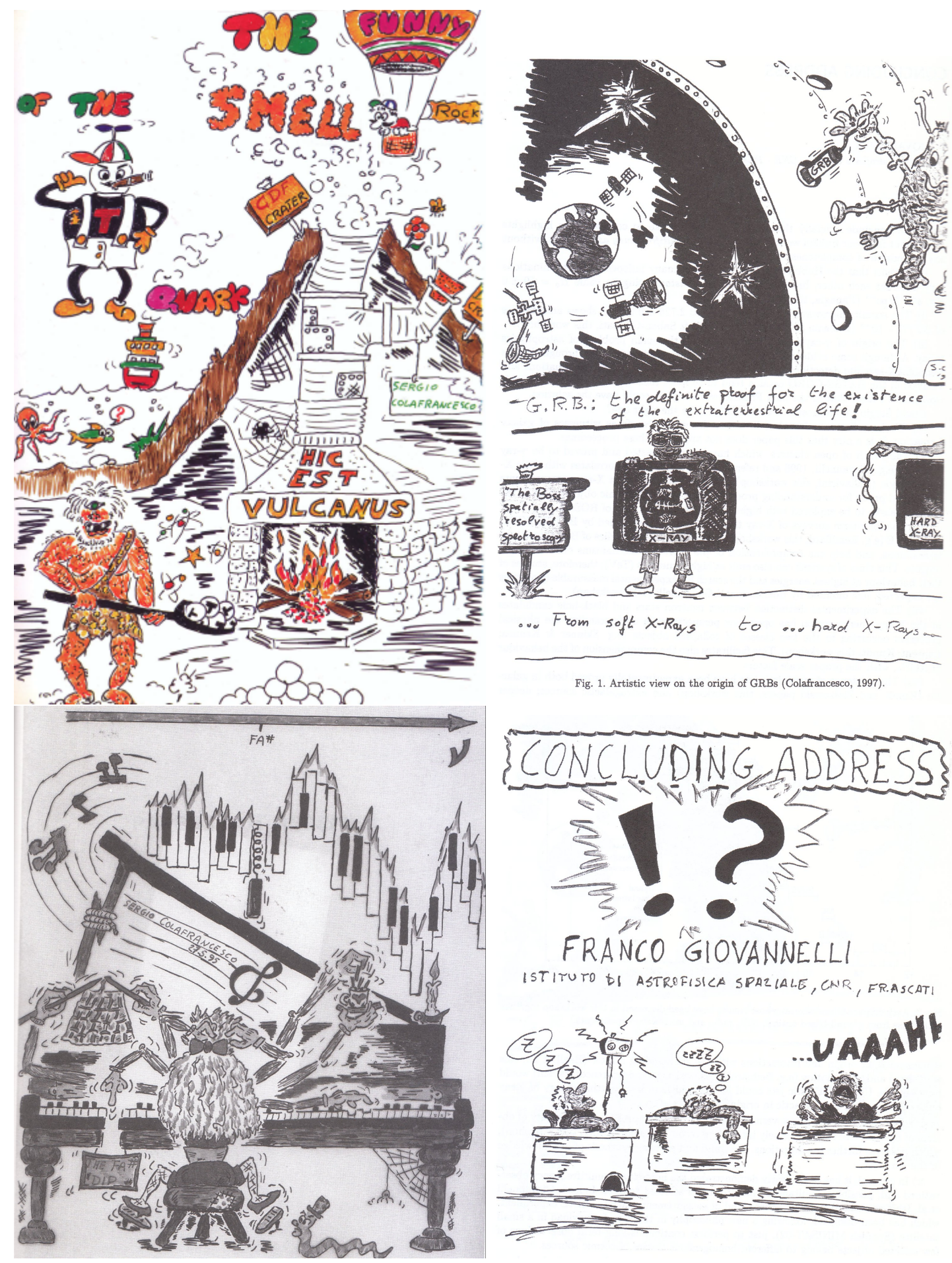

Figure 13: Left upper panel: Vulcano as seen by Sergio (Vulcano Workshop 1994). Right upper panel: The origin of GRBs as imagined by Sergio (Frascati Workshop 1997). Left lower panel: The pianist Paola Ghetti playing a fortepiano with a lack of one key in Lipari Greek theatre (Frascati Workshop 1995). Right lower panel. Concluding address by Franco Giovannelli as seen by Sergio (Frascati Workshop 1999). 


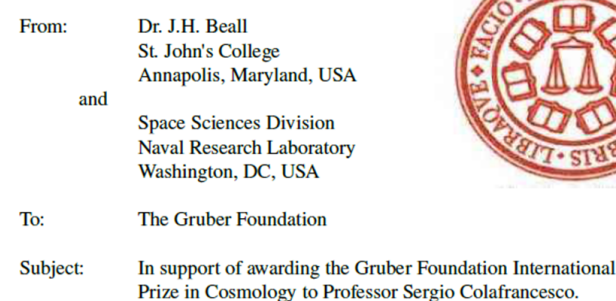

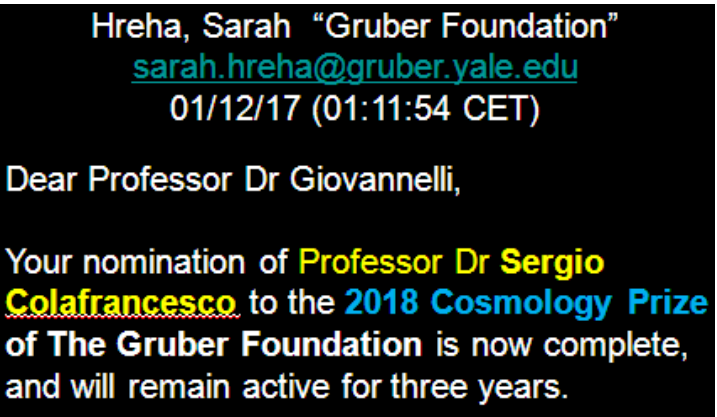

Figure 14: Left panel: James Howarth Beall's letter for supporting Sergio's candidature for the Grüber Price for Cosmology 2018. Right panel: Answer to the proposer Franco Giovannelli of Grüber Foundation.

These questions are at the crossroad of cosmology and astroparticle physics, and are milestones of the requirements from science funding agencies for new experiments and projects. The attempt to answer these questions will have the capacity to unlock some of the most crucial physical phenomena in the Universe and make a transition from the current blossoming of new ideas and experimental efforts to a new, cosmological paradigm for the origin and evolution of the universe. This can be sketched in Fig. 15.

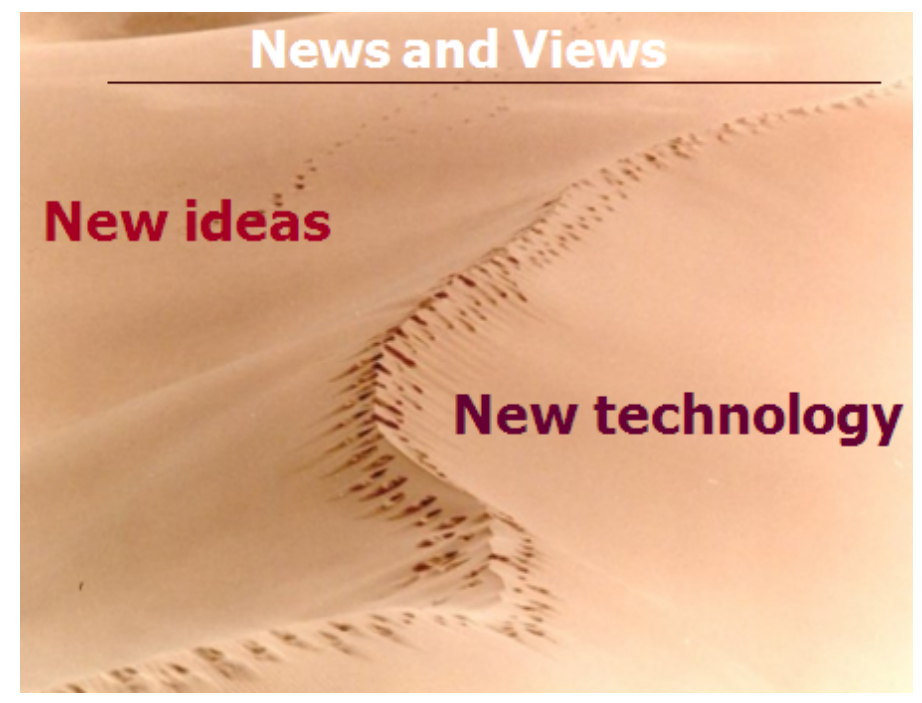

Figure 15: Sergio's last message about the progress in cosmology.

\section{Photo Gallery}

In my opinion Sergio can be considered as a Volcanic Scientist for his numerous activities. It is my desire to paint him as in Fig. 16.

In order to remember Sergio during our happy meetings, full of science and friendship, developed in Vulcano till 2011 and later in Mondello, I have collected some images among the many available, shown in Fig. 17. This is simply a dear memory of the great scientist and friend, Sergio. 


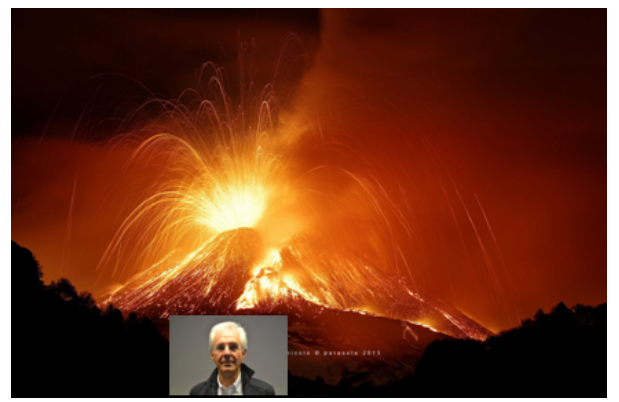

Figure 16: Sergio, the Volcanic Scientist.

One of the great ideas of Sergio - the caricaturist - was about the dark matter annihilation, reproduced in the Fig. 18.

During the commemoration of Sergio at Wits University on June 14, 2019, the actress Flavia Giovannelli - who knew Sergio at our workshops since 1990's - wanted to dedicate to him a wonderful poem by Eugenio Montale that seems written especially for him. In the following the Italian text of the poem and the English translation.

\section{Noi non sappiamo quale sortiremo}

Noi non sappiamo quale sortiremo domani, oscuro o lieto;

forse il nostro cammino a non tocche radure ci addurrà dove mormori eterna l'acqua di giovinezza; o sarà forse un discendere fino al vallo estremo, nel buio, perso il ricordo del mattino.

Ancora terre straniere forse ci accoglieranno: smarriremo la memoria del sole, dalla mente ci cadrà il tintinnare delle rime.

Oh la favola onde s'esprime la nostra vita, repente si cangerà nella cupa storia che non si racconta! Pur di una cosa ci affidi, padre, e questa è: che un poco del tuo dono sia passato per sempre nelle sillabe che rechiamo con noi, api ronzanti. Lontani andremo e serberemo un'eco della tua voce, come si ricorda del sole l'erba grigia nelle corti scurite, tra le case. 


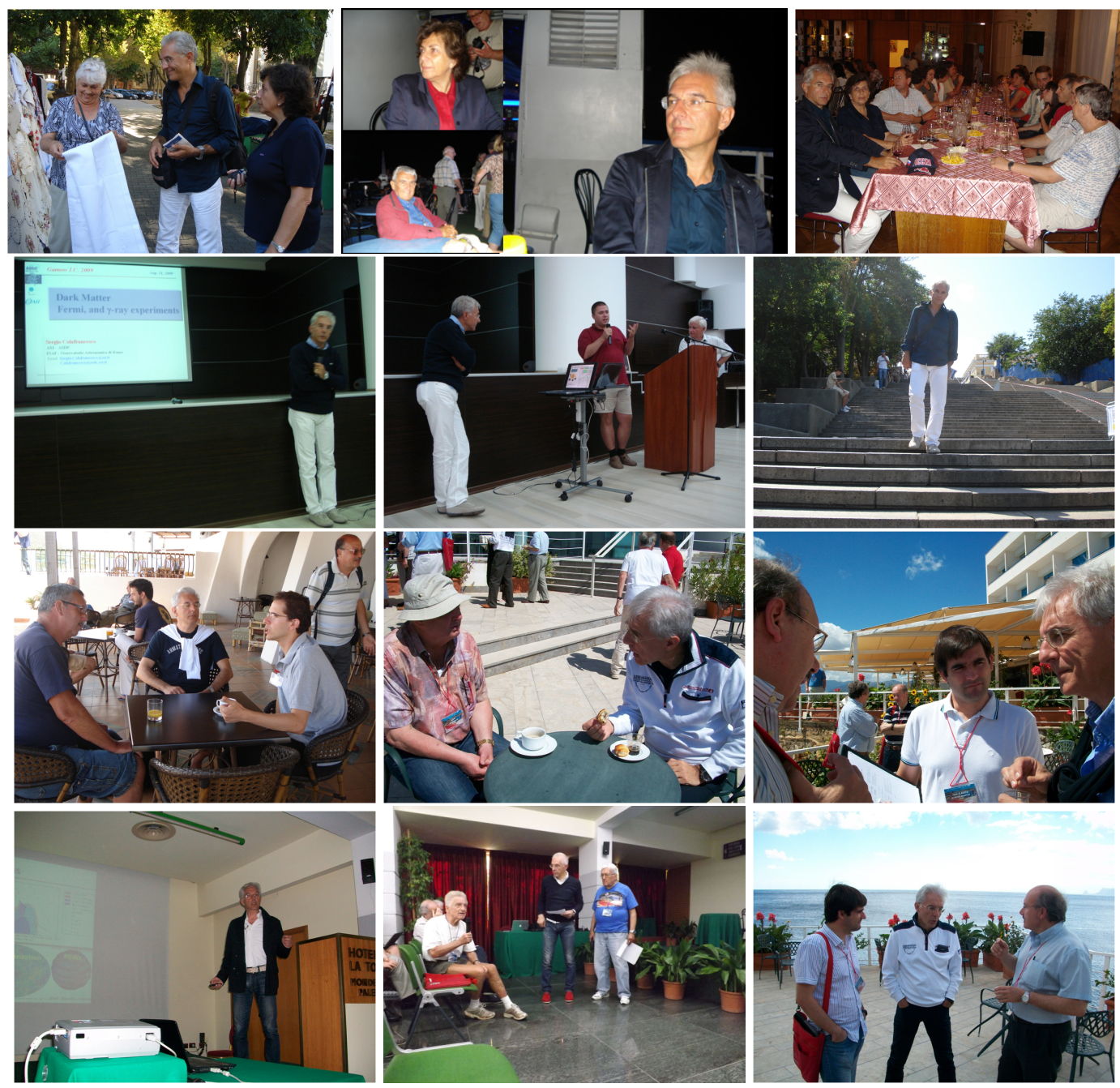

Figure 17: First line - Gamow Conference 2009 from left to right: Sergio and Lola in the market; Sergio, Franco and Lola during the excursion on a boat; the official dinner. Second line: Sergio's talk; Discussion after Sergio's talk; Sergio descends from the famous flight of steps immortalized by the film Bronenosjez Patjomkin (Battleship Potemkin) of Sergej Michajlovic Ejzenštejn (Credit: Franco Giovannelli, 2009). Third line - first from left: discussion during one coffee break of the Vulcano Workshop 2008; second and third: discussions during one coffee break of the Frascati Workshop 2013. Fourth line - first and second: Sergio's talk and Sergio as chairperson at the Frascati Workshop 2013; third: Sergio's typical posture when discussing with colleagues in Mondello at the Frascati Workshop 2013.

E un giorno queste parole senza rumore

che teco educammo nutrite

di stanchezze e di silenzi,

parranno a un fraterno cuore

sapide di sale greco.

\section{Eugenio Montale}




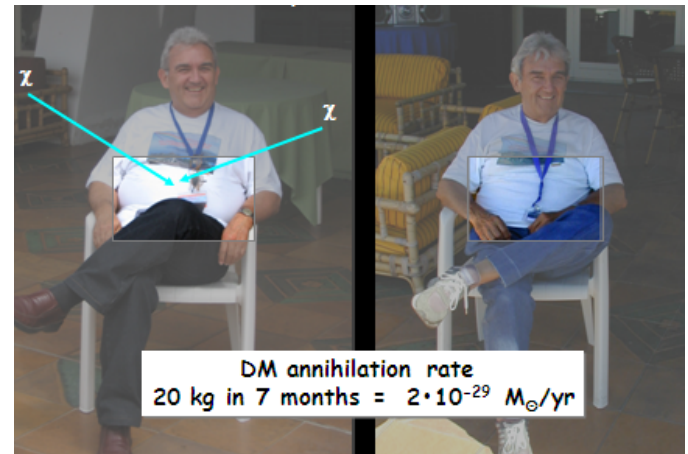

Figure 18: Sergio's vision of dark matter annihilation in Franco' stomach (Vulcano Workshop 2008).

\section{We do not know how we will turn out}

We do not know how we will turn out tomorrow, somber or happy;

perhaps our journey

skirting the clearings will bring us

to where the fountain of youth murmurs forever;

or maybe it will be a descent

until the final rampart,

in the dark, the memory of morning lost.

Further foreign lands

will perhaps welcome us: we will lose

the memory of the sun, from our mind

the tinkling of rhymes will fall.

$\mathrm{Oh}$, the fairy tale where our life

is expressed, suddenly

will transform into the dreary story that is not told!

There is one thing you can trust,

father, and this is it: that a little of your gift

would be passed forever in the syllables

that we carry with us, buzzing bees.

We'll go far away and keep an echo

of your voice, as the grey grass

remembers the sun

in the dark courtyards, between the houses.

And one day these words without sound

that learnt with you nourished

by tiredness and silence,

they will seem to a sympathetic heart

savoury of Attic salt.

\section{Eugenio Montale}


I would like to conclude this roundup of memories with a photograph, shown in Fig. 19, taken during the Frascati Workshop 2013, where on the terrace of the Splendid Hotel La Torre, Sergio and Giulio Auriemma (who also passed away in 2018) are portrayed in their splendor.

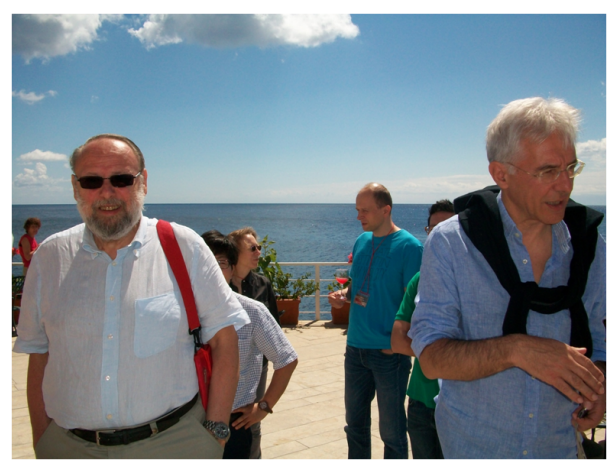

Figure 19: Giulio Auriemma and Sergio Colafrancesco during a coffee break of the Frascati Workshop 2013 on the terrace of the Splendid Hotel La Torre.

In the name of all the friends with whom we have shared many scientific adventures during our workshops, I would like to greet Sergio with an image that characterizes him completely (Fig. 20).

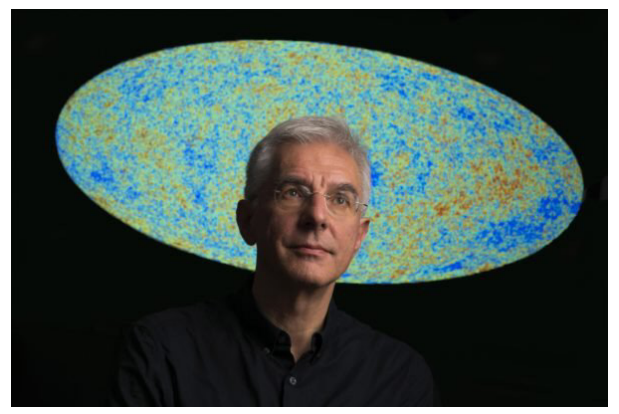

Figure 20: Sergio Colafrancesco with his preferred scientific background.

\section{Dear Sergio rest in peace.}

\section{Acknowledgments}

It is with great pleasure that I want to thank the referee, Dr James Howarth Beall (Jim), for his help in improving the text of this commemoration.

This research has made use of "The NASA's Astrophysics Data System".

\section{References}

[1] Ade, P.A.R. et al. (Planck Collaboration): 2013a, A\&A 554, id. A140, 19 pp.

[2] Ade, P.A.R. et al. (Planck Collaboration): 2013b, A\&A 557, id. A52, 17 pp. 
[3] Beall, J.H., Guillory, J., Rose, D.R., Schindler, S., Colafrancesco, S.: 2003, in Multifrequency Behaviour of High Energy Cosmic Sources - V, Franco Giovannelli \& Lola Sabau-Graziati (Eds.), ChJA\&A 3, Suppl., 137-146.

[4] Cavaliere, A., Colafrancesco, S.: 1988, ApJ 331, 660-671.

[5] Colafrancesco, S.: 2003, in Frontier Objects in Astrophysics and Particle Physics - IX, Franco Giovannelli \& Giampaolo Mannocchi (Eds.), SIF 85, 763.

[6] Colafrancesco, S.: 2004, Mem. SAIt. Suppl. 5, 432-437.

[7] Colafrancesco, S.: 2013, Acta Polytechnica CTU 53 Suppl., 560-572.

[8] Colafrancesco, S.: 2014, Acta Polytechnica CTU Proceedings 1 (1), 326-328.

[9] Colafrancesco, S., Lucchin, F., Matarrese, S.: 1989, ApJ 345, 3-11.

[10] Colafrancesco, S.: 2002, in Multifrequency Behaviour of High Energy Cosmic Sources - IV, Franco Giovannelli \& Lola Sabau-Graziati (Eds.), Mem. SAIt. 73, 1257-1258.

[11] Colafrancesco, S., Dar, A., De Rújula, A.: 2004, A\&A 413, 441-452. 